\title{
FURTHER OBSERVATIONS ON A RAPID METHOD OF PNEUMOCOCCUS TYPING
}

\author{
WADE W. OLIVER \\ From the Department of Bacteriology, Hoagland Laboratory, The Lang Island College \\ Hospital, Brooklyn, New York
}

This report is concerned with the experience obtained in a series of 100 consecutive, unselected cases of pneumonia in which typing of the pneumococcus from the sputum was made by (1) the rapid method described by the writer, ${ }^{1}(2)$ the Avery method, ${ }^{2}(3)$ a modified Avery method in which inulin is substituted for dextrose and an indicator is added to the medium, and (4) the mouse method, whenever possible.

\section{METHODS}

Rapid Method ${ }^{1}$.- The rapid method I have employed is a rapid precipitin test on bile treated sputum, based on the solubility of the pneumococcus in bile. After a direct smear of the sputum has been made, from 1 to $2 \mathrm{cc}$ of sputum are placed in a clean centrifuge tube. To the sputum is then added from 3 to 5 drops of undiluted ox bile (or a $10 \%$ solution of sodium taurocholate) and a sufficient quantity of sterile physiologic sodium chloride solution, if necessary, to insure a specimen of sufficient fluidity to allow of centrifugation. The mixture is then thoroughly stirred and broken up with a glass rod. It is sometimes advantageous to effect the breaking up and mixing of the sputum, bile and salt solution by grinding in a small mortar with a pestle. The tube is then heated in a water bath at a temperature of from 42 to $45 \mathrm{C}$. for 20 minutes, which time suffices for a solution of the pneumococci by the bile. The fluid is then centrifugalized. Of the centrifugate, from 0.3 to $0.5 \mathrm{cc}$ quantities are carefully pipetted into each of 3 small, scrupulously clean tubes. To the first tube is added from 1 to 2 drops of undiluted type 1 pneumococcus antiserum, and to the second and third tubes the same quantity of type 2 and type 3 antiserum, respectively. A positive precipitin test is evidenced by an almost immediate clouding and flocculation, which is enhanced by heating at $42 \mathrm{C}$. in a water bath for from 10 to 20 minutes.

Received for publication June 16, 1921.

1 Jour. Infect. Dis., 1920, 27, p. 310.

2 Jour. Amer. Med. Assn., 1918, 70, p. 17. 
Modified Avery Method.-The rapid cultural method for the determination of types of pneumococcus, described by Avery, ${ }^{2}$ consists in the use of a meat infusion broth, 0.3 to 0.5 acid to phenolphthalein, with $1 \%$ glucose and $5 \%$ rabbit blood. Recently, we have found that the substitution of $1 \%$ inulin for the dextrose offers certain advantages. In addition to the $1 \%$ inulin, Andrade indicator is added to the blood or ascitic fluid broth, $\mathrm{P}_{\mathrm{H}}$ 7.6.

Dextrose is broken down by both the streptococcus and pneumococcus. Inulin is attacked, with acid production, by the pneumococcus, whereas the streptococcus, with the exception of rare strains, fails to break down inulin. The presence of a fermentable carbohydrate in the medium appears to favor the growth of the pneumococcus, to the detriment of associated streptococci. If a pneumococcus is present in the sputum inoculated in the inulin Avery tube, the broth appears pink within from 4 to 5 hours at $37.5 \mathrm{C}$., and on longer incubation the color changes to a marked red. Incubation in the water bath, as suggested by Avery, hastens the growth of the pneumococcus, probably due to the more rapid heating of the contents of the tube to body temperature. The fact that such tubes, heated to $37.5 \mathrm{C}$. in a water bath, then quickly transferred to an ordinary incubator regulated to body temperature, yield as rapid a growth of pneumococci as do tubes incubated continuously in the water bath, would seem to bear out this contention.

When ascitic fluid is employed instead of blood, centrifugation is not necessary, prior to performing the agglutination and precipitin test. A small amount of loosely fibered cotton pushed carefully down through the broth to the bottom of the tube anchors gross particles of sputum and spontaneously agglutinated micro-organisms and yields a homogeneous supernatent bacterial suspension which can then be pipetted or even carefully poured into agglutination tubes.

SUMMARY OF FINDINGS BY TYPES

Of the 100 cases on which this communication is based, the following percentage of pneumococcus types was obtained:

TABLE 1

Percentage of Preumococcus types

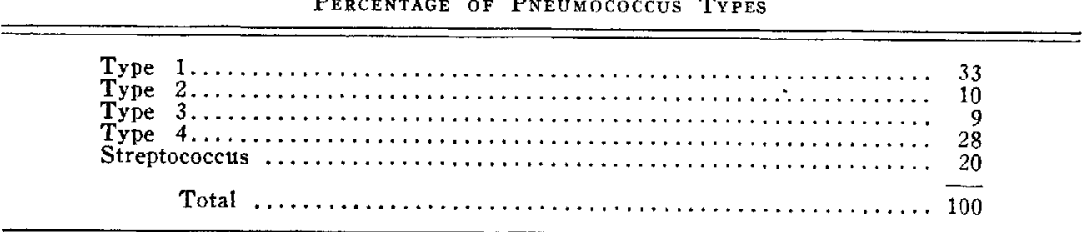


In each instance, the result obtained by the rapid precipitin method was checked by the Avery method. In addition, 5 of the 33 type 1 cases were checked by the mouse method and 2 by blood culture, 5 of the 10 type 2 cases by the mouse method and 1 by blood culture, 2 of the 9 type 3 by the mouse method and 1 by blood culture, 11 of the 28 type 4 by the mouse method, and 6 of the 20 streptococcus by the mouse method. In brief, in $33 \%$ of the 100 cases, comprising all of the various types of pneumococci and the streptococcus, the mouse method or blood culture or both were employed as a check on the results obtained by the rapid precipitin method and the Avery method. The reason for the fact that only 29 cases of the series were checked by mouse inoculation was the extreme paucity of mice at the time this work was being done.

TABLE 2

Degree of Intensity of the Rapid Precipitin Test

\begin{tabular}{|c|c|c|c|}
\hline 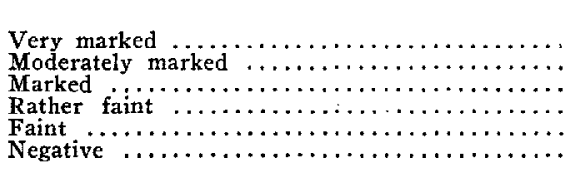 & 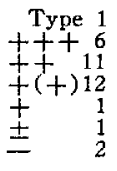 & $\begin{array}{l}\text { Type } 2 \\
+++6 \\
+1+2 \\
+(+) 2\end{array}$ & $\begin{array}{l}\text { Type } 3 \\
+++7 \\
++\quad 2\end{array}$ \\
\hline$\ldots \ldots \ldots \ldots \ldots$ & 33 & $\overline{10}$ & $\overline{9}$ \\
\hline
\end{tabular}

Of the 33 cases of type 1 infection, a positive type 1 test was obtained by the rapid precipitin method in 31 ; in 2 cases, the rapid precipitin method gave a negative result. In both of these cases, direct smears of the sputum revealed scattered pneumococci and large numbers of streptococci and Micrococcus catarrhalis. The 2 type 1 failures by the rapid method, in both of which the dextrose Avery and the inulin Avery were type 1 positive, as well as a blood culture in one case, were the only instances in which the rapid method failed. In the remaining 98 cases, the findings obtained by the rapid method agreed with the results obtained by the standard Avery or inulin Avery and the mouse method. This gives, in the series of 100 unselected cases, a $2 \%$ failure for the rapid precipitin method. In all of the other cases in which the rapid method was negative, the Avery or mouse method failed to reveal a type 1,2 , or 3 pneumococcus and showed either a type 4 pneumococcus or a streptococcus or both. Conversely, in no instance was a positive test obtained by the rapid method which failed of confirmation by the Avery or mouse method. 
The dextrose Avery method failed in 4 cases of the series, 3 of these being type 1 infections and 1 a type 2 infection. In all of the 3 type 1 failures by the standard Avery, the rapid precipitin method was type 1 positive (once ++ and twice $+[t]$ ), and in each instance the mouse method revealed a type 1 pneumococcus. In the type 2 failure with the dextrose Avery, the rapid precipitin method was $+(+)$ positive for type 2 , and the inulin Avery and mouse method were type 2 positive.

The inulin Avery method failed in one instance, a type 1 infection in which the dextrose Avery method also failed. In this case, the rapid precipitin method yielded $\mathbf{a}++$ test for type 1 , and the mouse method was type 1 positive.

\section{DISCUSSION}

In the original communication, ${ }^{1}$ reference was made to the fact that when a positive precipitin test is obtained, a clouding occurs in the fluid almost immediately on the addition of the specific antiserum. This clouding is not a homogeneous one, for when the tube is examined over a powerful electric light, more or less fine flocculi are visible within several minutes after the addition of the antiserum. This flocculation is hastened if the tube is immersed for from 10 to 20 minutes in water at $42 \mathrm{C}$. After this incubation, if the tubes are allowed to stand in the icebox for several hours, sedimentation occurs.

At present, work is being pursued on a rapid method of pneumococcus typing that will be applicable to small amounts of sputum. Such a method is especially desirable for cases of pneumonia in young children and for persons critically ill, from whom, often, only small quantities of sputum can be obtained. In two instances, in which only a small fragment of sputum was available, a micro-rapid precipitin test was used. The fragment of sputum was thoroughly emtlsified on a clean glass slide in 3 drops of bile diluted with sterile salt solution to $1: 10$. The mixture was then carefully drawn up into a fine capillary pipet, and the tip of the pipet was sealed in a fine flame. The capillary was then immersed in water at $42 \mathrm{C}$. for 20 minutes, after which centrifugation was employed. Then 4 small drops of the clear supernatent fluid were placed in a row on a clean glass slide. To the first 3 drops were added a tiny drop of type 1, 2 and 3 pneumococcus antiserum, respectively, and thorough admixture was effected. In one instance a type 2 reaction was obtained and in the other case a type 3 . In both instances, a finely flocculent clouding appeared in the respective drop 
within 30 seconds, the clouding being readily apparent to the naked eye when the slide was held up to an electric light, as well as being easily visible under the low power of the microscope. In each instance, the 3 remaining drops remained clear until after about 10 minutes, when the drops had so far concentrated as to deposit a white precipitate around the edge. The type 2 micro test was subsequently confirmed by the dextrose Avery method and the mouse method, and the type 3 micro test was confirmed by the dextrose Avery method.

A somewhat more promising way appears to be the use of a $0.5 \%$ washed agar which is allowed to solidify in a column in small, narrow agglutination tubes. In the test, 4 such tubes of agar are employed. In the first 3 tubes the top of the agar column is layered with a drop of sputum to which bile has been added. In the fourth tube, which serves as a control, the agar surface is covered with a drop of untreated sputum. The tubes are inmersed in a water bath at $42 \mathrm{C}$. for 20 minutes, which is a sufficient length of time for a solution of the pneumococci by the bile and an adsorption of precipitinogen by the agar surface. The sputum is then carefully washed and pipetted off the agar column with physiologic salt solution, following which several drops of the 3 type pneumococcus antiserums, respectively, are pipetted over the agar column in the respective first 3 tubes. As far as the work has progressed, it appears that when a positive test is obtained, say for a type 1 pneumococcus, the type 1 antiserum lying on the agar column, exhibits an almost instantaneous clouding if the tube is slightly shaken, whereas tubes 2 and 3 exhibit a layer of clear serum. In several instances, on incubating the tubes in a water bath at $42 \mathrm{C}$. for from 1 to 2 hours a ring test has developed below the surface of the agar column. Although by no means affording conclusive evidence, these results seem to suggest that the precipitinogen diffuses relatively rapidly into the agar, whereas the precipitin has a slower rate of diffusion. Experiments are in progress to determine the effect of layering columns of agar, into which the respective antiserums have been incorporated, with bile treated sputum.

In conclusion, I may say I am by no means convinced that any conclusions can rightfully be drawn from the $4 \%$ failure by the dextrose Avery method and the $2 \%$ failure by the rapid precipitin method in the present series of 100 cases. It is conceivable that, in another series, the ratio of failures might be reversed. Moreover, a much larger series 
than the one reported here would be required before any adequate conclusions could be drawn in regard to the comparative value of the standard dextrose Avery as compared with the inulin Avery. Theoretically and practically, so far as the present limited series goes, the modified Avery, containing inulin and an indicator, possesses certain advantages. One point of convenience lies in the fact that tubes which fail to show a red color after from 5 to 8 hours' incubation at $37.5 \mathrm{C}$. can safely be considered to be negative for pneumococci.

Finally, the rapid precipitin method has simply been advanced with the idea that, when it reveals a type 1 infection, it means a saving of from 5 to 8 hours in the administration of serum, a saving which, in certain cases, would seem to spell the difference between life and death.

\section{SUM MARY}

In 100 consecutive, unselected cases of pneumonia, in all but two instances, a typing of the pneumococcus has been effected within from 30 to 40 minutes by the rapid precipitin method, which was checked by the longer cultural or mouse method.

The substitution of inulin for dextrose and the addition of Andrade indicator seems to possess certain advantages over the standard Avery cultural method. 